\title{
Point of Care Ultrasound During the Covid-19 Pandemic Should Screen for Inter-Atrial Shunt
}

\author{
Rajkumar Rajendram, FRCP (Lond) ${ }^{a, c, d, *}$, Naveed Mahmood, FRCP (Edin) ${ }^{a-c}$, Ghulam Abbas Kharal, MD, MPH ${ }^{e}$, \\ Mubashar Kharal, MD ${ }^{a, c}$, Rishi Puri, MBBS, PhD, FRACP ${ }^{f}$ \\ ${ }^{a}$ Departments of Medicine and ${ }^{b}$ Cardiology, King Abdulaziz Medical City, King Abdulaziz International Medical Research Center, \\ Ministry of National Guard - Health Affairs, Riyadh, Saudi Arabia; ${ }^{c}$ College of Medicine, King Saud bin Abdulaziz University of \\ Health Sciences, Riyadh, Saudi Arabia; ${ }^{d}$ Departments of Anaesthesia and Intensive Care, Stoke Mandeville Hospital, Aylesbury, \\ Buckinghamshire, United Kingdom; ${ }^{e}$ Division of Cerebrovascular Disorders, Cleveland Clinic Neurological Institute, Cleveland Clinic \\ Foundation, Ohio, USA; ${ }^{f}$ Department of Cardiovascular Medicine, Heart \& Vascular Institute, Cleveland Clinic, Ohio, USA
}

Received June 1, 2020; revision received June 25, 2020; accepted June 27, 2020.

Advanced Ultrasound in Diagnosis and Therapy 2020;02:155-156

DOI: $10.37015 / A U D T .2020 .200049$

$\mathrm{T}$

The Coronavirus disease 2019 (Covid-19) has shone a spotlight on point of care ultrasound (POCUS) However, an investigation should only be performed if it will change management. We therefore read the expert consensus on critical care ultrasound applications [1] and review of the applications of critical care ultrasonography for covid-19 with great interest [2]. These reviews are particularly important as other specialist societies suggest that formal ultrasound and echocardiography performed by sonographers and radiologists should be limited [3]. Guidance on management of structural heart disease $[4,5]$ during the Covid-19 pandemic must be considered. These recommendations suggest that closure of inter-atrial defects (i.e. patent foramen ovale [PFO] and atrial septal defects [ASD]) should be deferred $[4,5]$.

So, clinicians may feel that screening for PFO is unnecessary during the Covid-19 pandemic. We agree that investigations must focus on identification of important abnormalities [3]. However, the incidence of venous thromboembolism, stroke, and systemic arterial embolization are high in patients with Covid-19.[6] Some of these thromboembolic phenomena may be due to paradoxical embolization via a PFO. So, patients with a PFO may be at high risk of systemic thromboembolism if they are infected with SARS-CoV-2. Deferring PFO closures is therefore counterproductive.
Whilst the only indication for PFO closure currently supported by randomized studies is secondary prevention after a PFO-related stroke [7]; treatment of hypoxia due to right-to-left (RTL) shunt is another important indication [7]. In this context the atypical nature of the 'acute respiratory distress syndrome' associated with Covid-19 is pertinent [8]. Occasionally, there is a mismatch between the severity of lung injury visible on imaging and the degree of hypoxia and shunt [8]. The aetiology of RTL shunt in Covid-19 is likely to be multifactorial. Whilst intrapulmonary shunt (IPS) will be present; Covid-19 may trigger extrapulmonary RTL shunt in select patients with an inter-atrial defect [8]. In these patients, RTL inter-atrial shunt (IAS) is likely to exacerbate hypoxia and worsen Covid-19. Determining the anatomical substrate for RTL shunt is required to determine the most appropriate management strategy. We therefore recommend screening for RTLIAS with bedside bubble contrast echocardiography.

Microbubble contrast $(10 \mathrm{ml})$ can be prepared by agitation of normal saline $(8 \mathrm{ml})$ with air $(1 \mathrm{ml})$ and, ideally, $1 \mathrm{ml}$ of the patient's blood between two Luer lock syringes connected via a 3 way stop cock [9]. When rapidly injected intravenously micro-bubbles within the saline should completely opacify the right heart [9]. This appears as a 'snowstorm' on 2D echocardiography (Fig. 1). The bubbles are normally filtered and disperse

\footnotetext{
* Correspondence Author: Department of Medicine, King Abdulaziz Medical City, Ministry of National Guard - Health Affairs, Riyadh, Saudi Arabia

e-mail:rajkumarrajendram@doctors.org.uk unrestricted use, distribution and reproduction in any medium provided that the original work is properly attributed.
} 
during passage through the pulmonary circulation. So any bubbles appearing in the left heart within 3 cardiac cycles confirm the presence of a right-to-left intracardiac shunt.[9] Bubbles appearing 'late' suggest that an intrapulmonary shunt is present [9]. Whilst the sensitivity transoesophageal echocardiography is greater than that of transthoracic echocardiography, this can be increased further if a Valsalva manoeuvre or positive end expiratory pressure is applied during administration of the microbubble contrast [9]. Although there are rarely any complications associated with this investigation, bubble contrast may cause transient worsening of hypoxia and so should not be used in patients with critical hypoxia.

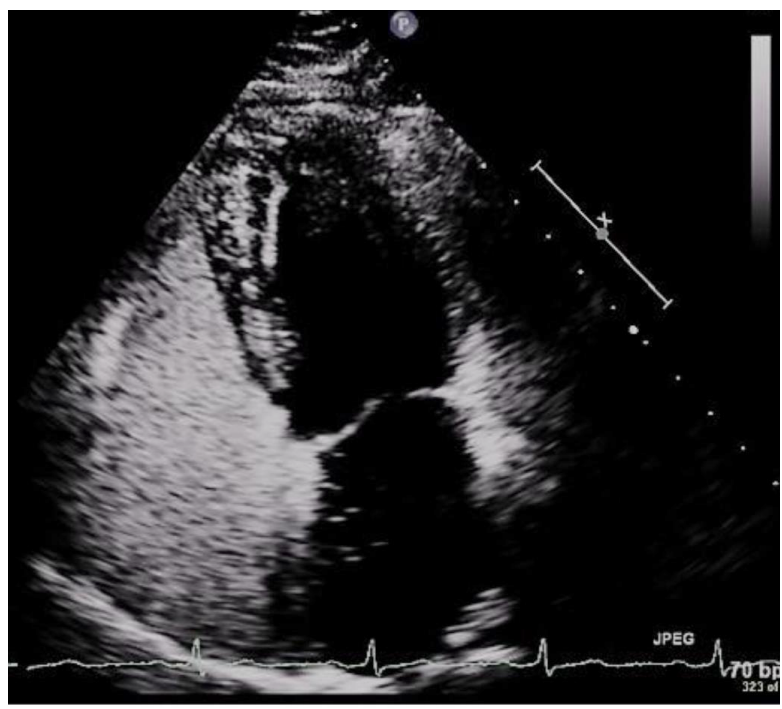

Figure 1 Microbubble contrast enhanced 2D echocardiogram. This microbubble contrast enhanced 2D echocardiogram shows opacification of the right atrium and right ventricle with saline microbubble contrast. Bubbles within the left atrium and ventricle, which in this case were present within 3 cardiac cycles, confirming the presence of an intracardiac shunt at the atrial level. The presence of bubbles within the left atrium after 3 cardiac cycles demonstrates the presence of an intrapulmonary shunt.

We recommend screening for an interatrial defect whenever:

1. There is a significant drop in the $\mathrm{PaO} 2 / \mathrm{FiO} 2$ ratio

2. A patient is more hypoxic than expected for the degree of lung injury identified

3. Hypoxaemia does not improve significantly with supplemental oxygen

4. Platypnea orthodeoxia is detected

Detection of RTLIAS will influence management, however, there are no randomized controlled clinical trials to guide these changes. Regardless. it most important to recognize that the standard approach to the management of refractory hypoxia, which aims to reduce
IPS, can exacerbate RTLIAS and may in fact worsen hypoxia.[8] Thus improving outcomes of these unusually hypoxic patients, requires treatment to reduce total shunt (i.e. IPS + EPS) by balancing the effects of any interventions on both IPS and EPS.

In select high risk patients with Covid-19, percutaneous closure of PFO could markedly improve hypoxia, reduce the need for invasive ventilation and prevent paradoxical embolization. Therefore, echocardiographic screening for ASD and PFO with bubble contrast is required in patients with Covid-19. This assessment can easily be performed by appropriately trained clinicians using POCUS.

\section{Conflict of Interest \\ none}

\section{References}

[1] Lv FQ, Wang JR, Yu X, Yang AP, Liu JB, Qian LX, et al. Chinese expert consensus on critical care ultrasound applications at COVID-19 pandemic. Advanced Ultrasound in Diagnosis and Therapy 2020; 4: 27-42.

[2] Liu X, Hai Y, Ma B, Chong W, Liu JB. Critical care ultrasonography and its application for COVID-19. Advanced Ultrasound in Diagnosis and Therapy 2020; 4: 43-49.

[3] Gackowski A, Lipczyń ska M, Lipiec P, Szyma ń ski P. Echocardiography during the coronavirus disease 2019 (COVID-19) pandemic: expert opinion of the Working Group on Echocardiography of the Polish Cardiac Society. Kardiol Pol 2020;78:357-363.

[4] Pło ń ska-Gościniak E, Suwalski P, Bartuś S, Kukulski T, Komar M, Wojakowski W, et al. Management of valvular and structural heart diseases during the COVID-19 pandemic: an expert opinion of the Working Group on Valvular Heart Diseases, the Working Group on Cardiac Surgery, and the Association of Cardiovascular Interventions of the Polish Cardiac Society. Kardiol Pol 2020; 78: 498-507. DOI: 10.33963/KP.15358.

[5] Welt FGP, Shah PB, Aronow HD, Bortnick AE, Henry TD, Sherwood MW, et al; American College of Cardiology' s Interventional Council and the Society for Cardiovascular Angiography and Interventions. Catheterization Laboratory Considerations During the Coronavirus (COVID-19) pandemic: from the ACC's interventional council and SCAI. J Am Coll Cardiol 2020;75:2372-2375.

[6] Klok FA, Kruip MJHA, van der Meer NJM, Arbous MS, Gommers DAMPJ, Kant KM, et al. Incidence of thrombotic complications in critically ill ICU patients with COVID-19. Thromb Res 2020; 191: 145-147.

[7] Collado FMS, Poulin MF, Murphy JJ, Jneid H, Kavinsky CJ. Patent foramen ovale closure for stroke prevention and other disorders. $J$ Am Heart Assoc 2018;7:e007146.

[8] Rajendram R, Kharal GA, Mahmood N, Puri R, Kharal M. Rethinking the respiratory paradigm of COVID-19: a 'hole' in the argument. Intensive Care Med 2020:1-2. DOI: 10.1007/s00134-02006102-6.

[9] Gallagher G, Joseph A, Rajendram R. Platypnea-orthodeoxia: Patent foramen ovale unmasked by pulmonary emboli. Indian J. Respir. Care 2018;7: 50-52. 